drawn from the early stage of larger study examining adolescent resilience. Adolescents were found to express eclectic and individual views surrounding the topic, with each response being uniquely representative of their beliefs. Themes commonly found included religion and God with many showing some degree of change. Some of these changes involved abandoning one religion in favor of another or changes in ideas about God. There was no significant relationship between those with a diagnosis and those without regarding their religious and spiritual beliefs; however, there were differences between groups in using beliefs to cope, beliefs about a spiritual force and its influence on day-to-day and world affairs. Spiritual and religious ideas are important for adolescents and those in the study showed a great diversity in ideas about this topic. It would be of further interest to research how these beliefs and ideas about religion and spiritual change over time and learn about how adolescents without a diagnosis use their beliefs to assist in coping.

\section{ECT and neuroprotection: a review and proposed study}

\section{Pigot, C Loo}

School of Psychiatry, University of New South Wales, Sydney, Australia

Background: Electroconvulsive therapy (ECT) is a highly effective treatment for depression but its use is limited by associated cognitive side-effects. Several theories have been proposed for the mechanisms underlying cognitive impairment with ECT: excitotoxic damage through influx of intracellular calcium or excessive glutamatergic transmission, alteration in opioid receptor density and induction of inflammatory processes (Krueger et al. 1992; Bazan et al. 2005). Several neuroprotective agents have been proposed to reduce ECT-related cognitive effects, leading to some trials in animal and human subjects.

Method: A comprehensive literature review (PubMed, Medline) identified animal and human clinical research trials of various agents proposed to have a neuroprotective effect on ECT-induced cognitive impairments.

Results: Several agents have been shown to reduce memory impairment after electroconvulsive shock (ECS) in rats, for example, calcium channel blockers, opioid receptor antagonists and glucocorticoid antagonists. However, results from clinical trials in humans have been less promising. There are early suggestions that ketamine anesthesia may be associated with fewer cognitive side-effects after ECT (McDaniels et al. 2006). Conclusions: Strong evidence from ECS models of neuroprotective strategies have to date not been confirmed in human clinical trials. However, clinical trials have been sparse, with small sample sizes and confounding methodological issues. We propose a randomized sham-controlled study to assess the effect of several neuroprotective agents on ECT-induced cognitive impairment.

\section{Spatial working memory task validation in a group of patients with schizophrenia and healthy volunteers}

\section{Piskulic ${ }^{1,2}, \mathrm{~J} \mathrm{Olver}^{1,2}, \mathrm{~K}$ Monshat ${ }^{2}$, T Norman $^{1,2}$, P Maruff ${ }^{3}$}

'The University of Melbourne, Melbourne, Australia; ${ }^{2}$ Austin Health; and

${ }^{3}$ CogState Ltd, Melbourne, Australia

Background: It is now agreed that cognitive deficit is an important aspect of the clinical presentation of schizophrenia. One parsimonious brain-behaviour model with good explanatory power for both animal and human models of the disorder is that of impairment of working memory (WM). Patients with schizophrenia show a reliable decrement of WM, which has been proposed to give rise to social and employment disabilities. The aim of the current study was to validate a computer-based behavioural spatial WM test in patients with schizophrenia.

Methods: A novel spatial WM test based on the ' $n$ back' paradigm was used to compare 15 medicated patients with schizophrenia against 15 healthy volunteers. The task involved remembering the position of a target card from an arrangement of between two and four cards presented in a radial configuration, with instructions to determine whether the card that was face up at the time of presentation was in the same location as a card that had turned face up on the previous trial (1-back condition) or two previous trials (2-back condition). All subjects were retested a week later to determine the stability and reliability of the test.

Results: Repeated-measures ANOVA showed that patients with schizophrenia performed worse than healthy controls on tests of spatial WM.

Conclusions: The behavioural $n$-back task proved to be a quick and reliable measure of spatial WM in patients with schizophrenia. Our data further support previous findings of spatial WM deficits in schizophrenia.

\section{Assessment of serotonin function, memory and spatial working memory using buspirone and placebo}

\footnotetext{
D Piskulic ${ }^{1,2}$, J Olver ${ }^{1,2}$, P Maruff ${ }^{3}$, T Norman ${ }^{1,2}$

'The University of Melbourne, Melbourne, Australia; ${ }^{2}$ Austin Health; and

${ }^{3}$ CogState Ltd, Melbourne, Australia
} 
Introduction: The neurotransmitters serotonin and dopamine are implicated in normal cognitive functioning. In healthy volunteers, dopamine depletion produces selective deficits of spatial working memory (SWM) while serotonin depletion results in deficits on a delayed word recall task (delayed verbal learning). Serotonin 5-HT1A receptors in man are found in postsynaptic locations in hippocampus and neocortex, and as somatodendritic autoreceptors in the raphe nuclei. 5-HT1A agonists have been shown to preferentially increase dopamine in the prefrontal cortex (PFC) acting by means of the 5-HT1A autoreceptors, which may influence cognitive function and memory in particular. We aimed to investigate the relationship between serotonin activity and memory performance in healthy volunteers.

Method: Ten male and 10 female healthy volunteers (aged between 18 and 60 years) were tested in a randomized, double-blind, crossover design receiving both buspirone $(20 \mathrm{mg})$ and placebo over the course of the study. All subjects completed tests of reaction time, SWM and verbal learning.

Results: Repeated-measures ANOVA showed significant decrement in delayed word recall performance after buspirone administration. SWM and RT data will be available for discussion at the time of presentation. Conclusions: Single dose of buspirone caused slight but nonetheless significant worsening in delayed verbal recall in healthy volunteers. This outcome may be because of 1) verbal memory decrements as a result of nondirect 5-HT1A receptor action on dopamine function in the PFC or, alternatively, 2) buspironeinduced changes in serotonergic function in the hippocampus.

\section{Altered neurophysiological parameters in an animal model of hyperactivity}

\section{TL Pitcher, JR Wickens, JNJ Reynolds}

University of Otago, New Zealand

Background: The spontaneously hypertensive rat (SHR) is commonly used as an animal model of attention deficit hyperactivity disorder-like behavioural characteristics. Little is known about the physiology of the SHR and its genetic control, Wistar-Kyoto (WKY). The aim of this research was to investigate the physiology of neurons in a brain area involved in movement initiation, the striatum.

Methods: Male rats from the Wistar (WI), SHR and WKY strains were anaesthetized with urethane $(1.8-2.4 \mathrm{~g} / \mathrm{kg})$. In vivo intracellular recordings were obtained using sharp microelectrodes from spiny projection neurons in the dorsal striatum. Cellular proper- ties were measured from electrophysiological records of spontaneous activity and neuronal responses to intracellular current injection.

Results: Recordings were made from 71 striatal spiny projection neurons (WI, $n=24$; SHR, $n=24$; WKY, $n=23$ ). Most cellular properties were similar across the three rat strains. However, two parameters of action potential firing differed between the SHR and WKY strains. (Current evoked action potential amplitude: SHR $76.5 \pm 7.5 \mathrm{mV}$, WKY $70.1 \pm 8.7 \mathrm{mV}$; duration: SHR $0.60 \pm 0.09 \mathrm{~ms}$, WKY $0.67 \pm 0.06 \mathrm{~ms} ; P<0.05$ one-way ANOVA.) The cellular properties of the WI strain were intermediate and not significantly different to either SHR or WKY.

Conclusions: The action potential parameter differences between the SHR and WKY strains may be related to the behavioural differences between the two strains. At the cellular level, this difference may also indicate that the two strains contain differences in membrane conductances in the striatal spiny projection neurons.

\section{Depression and health risk factors in the North West Adelaide Health Study \\ N Potts', D Wilson'2, A Taylor ${ }^{3}$, T Gill', G Schrader ${ }^{1,4}$, R Ruffin ${ }^{2}$}

'University of Adelaide; ${ }^{2}$ Health Observatory, Department of Medicine, The Queen

Elizabeth Hospital; 3 Population Research and Outcome Studies Unit,

South Australian Department of Health; and ${ }^{4}$ Department of Psychiatry,

The Queen Elizabeth Hospital, Adelaide, Australia

Background: The North West Adelaide Health Study is a population-based biomedical cohort study investigating the prevalence of chronic conditions and health-related risk factors. This study is based on a randomly selected group of individuals over the age of 18 , who agreed to be involved in a longitudinal health study.

Methods: Over the past 18 months, 3488 participants were assessed. General demographic and socioeconomic data were collected. Participants were assessed for the presence of a number of chronic conditions including major depression, chronic lung disease, quality of life using the SF-36 and health risk factors such as body mass index, smoking and exercise.

Results: Of the 3488 participants, $12.5 \%$ (436/3488) met criteria for depression. Depression was statistically significantly more likely among those who were current smokers, obese, had a high waist-hip ratio or waist circumference than those without these risk factors but less likely in those who undertook exercise. Depression was also associated with significantly lower scores on all the quality of life scores as 